\title{
Métodos en el estudio del pensamiento analógico: tradiciones y nuevas perspectivas
}

Olguín, M.V.*valitao@yahoo.es

Tavernini, L.M.** micaelatavernini@gmail.com

Gómez, A.E. ${ }^{* * * 1}$ ana.e.gz@hotmail.com

\section{Resumen}

El objetivo del presente trabajo fue revisar los métodos de investigación utilizados en los últimos años en el estudio del subproceso cognitivo de recuperación de análogos desde la memoria de largo plazo en el razonamiento por analogía. En primer lugar se analizaron los modos de trabajo propios de los estudios experimentales tradicionales que lograron alto grado de control y rigurosidad, pero que carecían de validez ecológica. A continuación, se desarrollan los programas de investigación de tipo naturalistas que buscaron abordar el fenómeno desde un enfoque diferente que evitó la artificialidad de los estímulos generados en los laboratorios de Psicología con la consecuente pérdida de control. Por último, se revisa una propuesta de trabajo más reciente, que busca superar las debilidades de ambos paradigmas y, a la vez, conjugar sus fortalezas, de manera que se puedan retomar los aspectos más valiosos de cada uno de ellos.

Palabras clave: razonamiento analógico- paradigmas tradicionales- paradigmas naturalistas.

\begin{abstract}
The aim of this study was to review the research methods used in recent years in the study of cognitive similar thread recovery from long-term memory in reasoning by analogy. First own work modes of traditional experimental studies that achieved high degree of control and rigorously analyzed, but lacking ecological validity. Then research programs such naturalists who sought to tackle the phenomenon from a different approach which avoided artificiality of stimuli generated in the laboratories of psychology with the consequent loss of control are developed. Finally, a proposal of more recent work, which seeks to overcome the weaknesses of both para-
\end{abstract}

* Olguín, María Valeria: es Doctora en Psicología, Magister en Psicología Cognitiva y Aprendizaje, Profesora en Psicología, Licenciada en Psicología; investigadora del CONICET, docente de la Universdad Nacional del Comahue.

** Tavernini, Lucía Micaela: es licenciada en psicología investigadora del CONICET, docente de la Fac. de Cs. De la Educación de la Universidad Nacional del Comahue.

*** Gómez, Ana: Psicóloga, Ayudante de primera en las cátedras de Metodología de la Investigación en Psicología I y II en la Universidad Nacional del Comahue, Ayudante de primera en la cátedra de Psicología Cognitiva I e Investigadora integrante del grupo de investigación "El rol de la semántica y de la pragmática en el pensamiento por analogía" 
digms and, in turn, combine their strengths, so that they can pick the most valuable aspects each is reviewed.

Keywords: analogical reasoning paradigms naturalists-traditional paradigms.

En numerosas actividades cotidianas, tales como argumentar, enseñar un concepto novedoso o resolver un problema, utilizamos con frecuencia un tipo de razonamiento inductivo llamado pensamiento analógico. (Gentner, Holyoak y Kokinov, 2001; Holyoak y Thagard, 1995) Este tipo de razonamiento implica advertir que dos situaciones se encuentran estructuradas por un sistema de relaciones y roles similar al sistema de relaciones y roles que estructura otra situación diferente. (Gentner, 1983; Holyoak, 1984) Así, los seres humanos podemos transferir conocimientos desde una situación más conocida (análogo base) hacia otra más novedosa, (análogo meta).

A veces, las situaciones que se comparan pertenecen a un mismo dominio de conocimiento, en este caso la analogía es denominada analogía intradominio. Es de esperar que si dos análogos provienen de un mismo dominio de conocimiento compartan similitudes en sus relaciones y en los objetos. (Trench y Minervino, en prensa) En cambio, cuando pertenecen a distintos dominios de conocimiento (i.e. analogías interdominio) los objetos y relaciones no se parecen en la misma medida que en las analogías intradominio.

Según Gentner y Smith (2012), las analogías varían ampliamente en su apariencia, contenido y uso, pero más allá de ello se caracterizan por un conjunto de procesos comunes. El primero de estos subprocesos es la recuperación de una representación almacenada en memoria de largo plazo a partir de otra representación actual presente en memoria de trabajo. Una vez que ambas situaciones se encuentran activas, tiene lugar el segundo subproceso que es el establecimiento de correspondencias, gracias al cual se alinean las representaciones base y meta. El último subproceso es la proyección de inferencias de un análogo al otro.

La historia de la investigación sobre el pensamiento analógico tiene sus principales antecedentes en la Psicología (e.g., Dorolle, 1949; Duncker, 1926; Herbart, 1898) y la Filosofía de la Ciencia. (e.g., Black, 1962; Hesse, 1966) El resurgimiento más reciente del interés por el tema puede ser localizado en los desarrollos de las Ciencias Cognitivas. Nuestro objetivo en el presente trabajo fue hacer una revisión de los métodos que han caracterizado los estudios sobre el subproceso de recuperación en el pensamiento analógico durante los últimos años. En primer lugar analizaremos los puntos fuertes y débiles de dos paradigmas metodológicos, y en segundo lugar presentaremos un enfoque que busca superar las debilidades de ambos paradigmas y, a la vez, conjugar sus fortalezas.

\section{Paradigma experimental tradicional}

A partir de la década del 80, dos teorías han dominado el estudio del pensamiento analógico: la Teoría de la Proyección de la Estructura, de Gentner y cols. (Gentner, 1983, 1989; Gentner y Smith, 2012) y la Teoría de las Múltiples Res- 
tricciones, de Holyoak y cols. (Holyoak y Thagard, 1989, 1995; Hummel y Holyoak, 1997) En ambos grupos de investigación se ha utilizado como método la experimentación de laboratorio para, posteriormente, generar simulaciones computacionales. (e.g., Falkenhainer, Forbus y Gentner, 1989; Holyoak y Thagard, 1989; Doumas y Hummel, 2012; Hummel y Holyoak, 1997, 2003)

Tal como sucede en muchos estudios sobre la memoria, el paradigma más utilizado para investigar la recuperación analógica comprende dos fases: una fase de codificación y una fase de recuperación. Durante la fase de codificación los participantes reciben un conjunto de historias entre las que se encuentra el análogo base, de aquí la denominación que le diera Dunbar (2001) de Paradigma de recepción. Durante la segunda fase se les presenta a los participantes una tarea meta para la cual la recuperación del análogo base resulta crítica. Los experimentadores evalúan de este modo el proceso por el cual un análogo meta provoca la recuperación del análogo base de memoria de largo plazo.

En un ejemplo paradigmático Gick y Holyoak (1980), pidieron a los participantes en la primera fase que leyeran un conjunto de historias entre las que se encontraba la historia de un militar que quería atacar con su tropa a un dictador en su fortaleza. Como los caminos se encontraban minados, el militar dividió su ejército en pequeños grupos, y tras ubicarlos sobre caminos diferentes, los hizo converger simultáneamente sobre la fortaleza. En una segunda fase, se les presentó a los participantes un problema meta que no mantenía similitudes superficiales con el análogo base, y versaba sobre un médico que debía destruir el tumor estomacal de un paciente. Un rayo intenso destruiría los tejidos sanos, y a baja intensidad no lograría destruir el tumor. El problema que los participantes debían resolver es cómo atacar el tumor con la intensidad apropiada. Los autores evaluaron, en qué medida, a partir del problema del tumor los participantes recuperaban la historia del militar, y transferían la solución.

Los resultados de este estudio mostraron que, a pesar de tenerlo disponible, las personas tienen dificultad para recuperar un caso análogo de su memoria si éste no mantiene similitudes superficiales con la situación que actualmente se tiene en mente. Es decir, las personas que leían en la primera fase la historia del militar, no la recuperaban cuando se les presentaba el problema del médico que se enfrenta al paciente con el tumor. En oposición, si el análogo base guardaba similitudes superficiales con el meta la recuperación resultaba sencilla. Esto fue estudiado por Keane (1987), quien utilizó como análogo base una historia de un paciente con un tumor cerebral, y como análogo meta la misma historia del paciente con tumor estomacal empleada por Gick y Holyoak. (1980)

Estos resultados generaron un fuerte impacto a dos niveles. Por un lado, a nivel teórico inició un conjunto de estudios interesados en las razones por las cuales la recuperación de información que no guarda similitudes superficiales resulta difícil para quien razona por analogía. Por otro lado, a nivel metodológico definió al paradigma de recepción como el método tradicional para el estudio de la recuperación. Una gran cantidad de estudios posteriores utilizaron este paradigma experimental introduciendo pequeñas variaciones en el tipo de materiales utilizados, las separaciones de tiempo y espacio entre las dos fases, etc. (para una revisión véase Trench y Minervino, en prensa) 
El método experimental, gracias al alto grado de control, permitió a los investigadores de la tradición descripta estudiar la relación entre variables "puras" sin contaminación de otras variables. Esto posibilitó establecer relaciones causales con precisión, confiriendo a estos estudios un alto poder predictivo. Sin embargo, en este método subyacía como debilidad la falta de validez ecológica, ya que los estímulos utilizados en los laboratorios resultaban artificiales a las personas. Quedaba así pendiente averiguar si lo observado dentro del laboratorio era válido fuera de él. En otras palabras, restaba estudiar las analogías que realizan las personas en escenarios naturales. (Gentner y Smith, 2012)

\section{Estudios Naturalistas}

A partir de los años 90, diversos autores comenzaron a realizar estudios con el fin de indagar el uso espontáneo de las analogías en diversos contextos del mundo real, tales como la enseñanza en el aula (e.g., Richland, Holyoak, y Stigler, 2004), la disertación facultativa (e.g., Saner y Schunn, 1999), la producción y exposición de conocimiento científico (e.g., Dunbar, 1997) y el discurso político. (e.g., Blanchette y Dunbar, 2001) La estructura metodológica general de estos estudios consistió en el análisis de analogías una vez que ya habían sido realizadas (i.e. ex post facto). En los estudios mencionados se registraron analogías, se las codificó cualitativamente y se las analizó cuantitativamente. A diferencia del paradigma experimental de recepción en que los investigadores proveían los análogos a los participantes, en los estudios naturalistas se utilizó un paradigma que Blanchette y Dunbar (2000) llamaron Paradigma de producción, ya que los análogos a ser recuperados provenían de la memoria autobiográfica de los participantes.

Un ejemplo paradigmático en el campo fue el estudio realizado por Blanchette y Dunbar (2001). En él, los autores analizaron los artículos aparecidos en periódicos de Montreal relativos al referendum sobre la independencia de Quebec de Canadá. En estos artículos, diferentes periodistas expresaban su posición recurriendo al uso de analogías. Los autores codificaron las analogías según su distancia semántica y encontraron que un elevado porcentaje de las analogías analizadas se caracterizaban por tener baja similitud superficial y pertenecer a dominios distantes (i.e., analogías interdominio).

Estos resultados fueron consistentes con los de otros estudios naturalistas (Blanchette y Dunbar, 2000; Dunbar, 1997; Richland, Holyoak, y Stigler, 2004; Saner y Schunn, 1999), y muestran un patrón opuesto al de los estudios experimentales en los cuales la recuperación fue fundamentalmente intradominio. Este fenómeno fue denominado por Dunbar (2001) como Paradoja analógica. La explicación que Dunbar le dio a estos resultados paradojales fue que en los contextos naturales las personas codifican las situaciones de un modo estructural, esto es, prestando atención a los aspectos centrales abstractos y dejando de lado las características superficiales. De manera opuesta, en el laboratorio solo se estimula un procesamiento superficial de las mismas.

Tanto los métodos experimentales como los naturalistas han contribuido al estudio de la recuperación en el pensamiento por analogía. Sin embargo, tal y como lo destacan Trench y Minervino (en prensa), ambos enfoques contienen puntos 
fuertes y débiles. Una de las fortalezas de los estudios naturalistas es que permiten a los investigadores estar en contacto con las variables tal como se comportan en la realidad, lo cual confiere a estos estudios una alta validez ecológica. Sin embargo, esto lo hace a expensas del control sobre el fenómeno observado, y con ello se reduce la validez interna. En el caso estudiado más arriba, Blanchette y Dunbar (2001) debieron enfrentar la crítica de que no lograron determinar si los análogos fueron efectivamente recuperados de la memoria o si las personas los inventaron ad hoc. (Trench, Oberholzer, Adrover y Minervino, 2009) Por otro lado, como expusimos en el apartado anterior, el control logrado por el método experimental a veces se alcanza a expensas de la validez ecológica.

\section{Nuevas perspectivas}

En un intento de subsanar algunas deficiencias, tanto de los estudios naturalistas como de los experimentales, Trench y Minervino (en prensa) se propusieron diseñar un procedimiento híbrido que les permitiera conservar la validez ecológica de los estudios naturalistas, sin perder la capacidad de predicción de los experimentos de laboratorio.

Los autores presentaron a los participantes situaciones meta análogas a cuatro películas populares (i.e., análogos base críticos): El secreto de sus ojos, Shrek, Spiderman y Jurassic Park. A un grupo de participantes se les presentó una situación meta superficialmente similar al análogo base crítico, mientras que a otro grupo se les presentó situaciones meta superficialmente diferentes. En la primera tarea los participantes tenían que generar argumentos analógicos a fin de disuadir a los personajes de la situación meta de llevar a cabo una acción que podría generar consecuencias negativas. Al igual que en el paradigma de producción, estas analogías debían ser recuperadas de la memoria autobiográfica ya que, según lo propuesto por autores como Dunbar (2001) y Hofstadter y Sander (2013), los análogos base que se codifican naturalmente reciben un procesamiento más abstracto y estructural que los que se codifican en contextos de laboratorio. El hecho de que la tarea meta fuese altamente realista, le confirió a este estudio una validez ecológica equiparable al paradigma de producción utilizado en los estudios naturalistas.

En una segunda tarea, se les preguntó a los participantes si habían recordado alguna película durante la recuperación de análogos base para argumentar, con el objetivo de determinar si habían recuperado el análogo base crítico correspondiente a una película popular. En caso afirmativo, debían responder preguntas sobre el mismo y finalmente establecer la analogía entre la película y la situación meta. Sólo aquellos casos en que los participantes fueron capaces de establecer correctamente la analogía entre la película y la situación meta recibieron análisis posterior. Este criterio permitió asegurar que el análogo base crítico efectivamente había sido recuperado de su memoria a largo plazo en lugar de ser inventado ad hoc, lo cual confirió al estudio una validez interna equiparable a la del paradigma de recepción utilizado en los experimentos de laboratorio.

Los resultados evidenciaron que la probabilidad de recuperación de análogos base críticos, a partir de análogos meta superficialmente similares, fue mucho mayor que la recuperación a partir de análogos meta superficialmente diferentes. El efecto 
hallado es consistente con los resultados de la tradición experimental. (e.g., Gick y Holyoak, 1980)

Los autores sostienen que los resultados hallados por Blanchette y Dunbar, (2001) no se explican por el hecho de que los diferentes contextos provoquen que la mente codifique la información de un modo más o menos abstracto, o que la recuperación tenga menos restricciones superficiales en contextos realistas. En cambio, sí podrían explicarse por un insuficiente control de variables como la invención ad hoc de análogos base. (Trench y Minervino, en prensa)

Nuestro objetivo en el presente trabajo, fue revisar los métodos utilizados en los últimos años en el estudio de la recuperación en el pensamiento analógico, analizando sus fortalezas y debilidades. Si bien quienes realizaron estudios experimentales tradicionales lograron estudiar el pensamiento analógico con alto grado de control y rigurosidad, reconocieron la necesidad de salir del laboratorio y observar las analogías que hacen las personas en su vida cotidiana. Esta necesidad fue retomada en recientes programas de investigación naturalista que movilizaron a la comunidad científica para abordar el fenómeno desde un enfoque diferente que evita la artificialidad de los estímulos generados en los laboratorios de Psicología.

El aporte de Trench y Minervino (en prensa), resulta, en nuestra opinión, positivo, ya que además de resolver una disquisición teórica sobre el peso de la distancia semántica en la recuperación de información de memoria de largo plazo, logró combinar el alto control de variables y la capacidad inferencial con el realismo propio de las tareas y escenarios naturalistas. Creemos que esto es alentador para generar estrategias que no disocien el laboratorio de los escenarios reales, sino que sinteticen los aspectos más valiosos de cada uno.

\section{BIBLIOGRÁFIA}

- Black, M. (1962). Models and Metaphors. Ithaca: Cornell University Press.

- Blanchette, I., y Dunbar, K. (2000). How analogies are generated: The roles of structural and superficial similarity. Memory and Cognition, 28, 108-124.

- Blanchette, I., y Dunbar, K. (2001). Analogy use in naturalistic settings: The influence of audience, emotion, and goals. Memory \& Cognition, 29, 730-735.

- Doumas, L.A.A., y Hummel, J.E. (2012). Computational models of higher cognition. En K. J. Holyoak y R. G. Morrison (Eds.), The Oxford Handbook of Thinking and Reasoning (pp. 52-66). New York: Oxford University Press.

— Dorolle, M. (1949). Le Raisonnement Par Analogie. París: Presses Universitaires. - Dunbar, K. (1997). How scientists think: Online creativity and conceptual change in science. In T. B. Ward, S. M. Smith, y S. Vaid (Eds.), Creative thought: An investigation on conceptual structures and processes (pp. 461-493). Washington, DC: APA Press.

- Dunbar, K. (2001). The analogical paradox: Why analogy is so easy in naturalistic settings, yet so difficult in the psychology laboratory? En D. Gentner, K. J. Holyoak y B. K. Kokinov (Eds.), The analogical mind: Perspectives from cognitive science (pp. 313-334). Cambridge, MA: The MIT Press. 
- Duncker, K. (1926). A qualitative (experimental and theoretical) study of productive thinking (solving of comprehensive problems). Journal of Genetic Psychology, 68, 97-116.

— Falkenhainer, B., Forbus, K. D., y Gentner, D. (1989). The structure-mapping engine: Algorithm and examples. Artificial Intelligence, 41, 1-63.

- Gentner, D. (1983). Structure-mapping: A theoretical framework for analogy. Cognitive Science, 7, 155-170.

- Gentner, D. (1989). The mechanisms of analogical transfer. En S. Vosniadou y A. Ortony (Eds.), Similarity and Analogical Reasoning (pp. 199-241). Cambridge, UK: Cambridge University Press.

- Gentner, D., Holyoak, K. J., y Kokinov, B. (Eds.). (2001). The analogical mind: Perspectives from cognitive science. Cambridge, MA: MIT Press.

— Gentner, D. y Smith, L. (2012). Analogical reasoning. En V. S. Ramachandran (Ed.), Encyclopedia of Human Behavior (2nd Ed.) (pp. 130-136). Oxford, UK: Elsevier.

— Gick, M. L., y Holyoak, K. J. (1980). Analogical problem solving. Cognitive Psychology, 12, 306-355.

- Herbart, J. H. (1898). Letters and lectures on education. London: Sonnenschein.

- Hesse, M. B. (1966). Models and analogies in science. Notre Dame, Ind.: University Press.

- Holyoak, K. J. (1984). Analogical thinking and human intelligence. Advances in the psychology of human intelligence, 2, 199-230.

- Holyoak, K. J., y Thagard, P. R. (1989). Analogical mapping by constraint satisfaction. Cognitive Science, 13, 295-355.

- Holyoak, K. J., y Thagard, P. R. (1995). Mental Leaps: Analogy in Creative Thought. Cambridge, MA: MIT Press.

- Hofstadter, D., y Sander, E. (2013). Surfaces and essences: Analogy as the fuel and fire of thinking. NewYork: Basic Books.

- Hummel, J. E., y Holyoak, K. J. (1997). Distributed representations of structure: A theory of analogical access and mapping. Psychological Review 104, 427-466.

- Hummel, J. E., y Holyoak, K. J. (2003). A symbolic-connectionist theory of relational inference and generalization. Psychological Review, 110, 220-264.

- Keane, M. (1987). On retrieving analogues when solving problems. The Quarterly Journal of Experimental Psychology, 39(1), 29-41.

— Richland, L. E., Holyoak, K. J., y Stigler, J. W. (2004). Analogy use in eighthgrade mathematics classrooms. Cognition and Instruction, 22, 37-60.

- Saner, L., y Schunn, C. D. (1999). Analogies out of the blue: When history seems to retell itself. In M. Hahn y S. Stoness (Eds.), Proceedings of the 21st Annual Conference of the Cognitive Science Society (pp. 619-624). Mahwah, NJ: Erlbaum.

- Trench, M., y Minervino, R. A. (en prensa). The role of surface similarity in analogical retrieval: Bridging the gap between the naturalistic and the experimental traditions. Cognitive science. doi: 10.1111/cogs.12201

- Trench, J. M., Oberholzer, M. N., Adrover, J. F. y Minervino, R. A. (2009). Recuperar análogos distantes es difícil: una puesta a prueba de la eficacia del paradigma de generación para promover recuperaciones interdominio. Psykhe, Revista de la Escuela de Psicología de la Pontificia Universidad Católica de Chile, 1, 39-48. 